\title{
PHYSIOLOGICAL ROLE OF PROSTAGLANDINS IN HUMAN PARTURITION
}

\section{Fuchs, F., New York.}

The ability of prostaglandin $E_{1}, E_{2}$ and $F_{2 \alpha}$ to stimulate the uterus to contract at any time of gestation and to interrupt pregnancy at any stage has led to a number of studies on their possible role in the physiological activation of the uterus during parturition. The hypothesis of an important role is supported by the fact that prostaglandin synthetase inhibitors can inhibit preterm labor and delay parturition. The marked rise in amniotic fluid levels of $\mathrm{PGE}_{2}$ and $P F_{2} \alpha$ during term labor and the rise in circulating levels of PGF metabolite (PGFM) during advanced labor also suggests a role for prostaglandins in the mechanism of labor. An important question is whether prostaglandins are involved in the initiation or maintenance of uterine contractions, or whether they are released as a consequence of labor and participate in other cellular functions.

A gradual rise in the excretion of PG metabolites during gestation indicates that the production increases. A marked rise following delivery attests to an increased production during labor, but no significant rise has been detected before the onset. All uterine tissues have the capacity to synthesize prostaglandins, although the spectrum varies somewhat from tissue to tissue, and no marked changes occur in this capacity at the onset of labor. It seems clear that the capacity to produce PGs from endogenous substrates exists long before labor begins, and that substrate availability is not a limiting factor. We must therefore assume that under in vivo conditions, prostanoid synthesis is kept at bay by inhibitory factors which are withdrawn during parturition, or that stimulatory factors increase the rate of production of PGs during labor.

We have found that oxytocin is a potent stimulator of prostaglandin synthesis. It is an attractive hypothesis that a fetal signal initiates labor, a belief held already by Hippocrates. We have proposed that fetal oxytocin provides such a signal by stimulation of prostanoid production in uterine tissues which then enhances the contractions elicited by the perhaps subthreshold levels of oxytocin reaching the myometrium. Vasopressin stimulates PG production in the adult kidney and fetal vasopressin may contribute to amniotic fluid prostaglandins by stimulating the fetal kidney production of PGE which is then secreted with fetal urine.

Vaginal examination, sweeping of the membranes, manipulation of the cervix, and rupture of the membranes all cause rapid and substantial elevations in amniotic fluid PGs and in the plasma level of PGFM. The lower pole of the membranes and the tissues around the cervical os may therefore be especially sensitive to tactile stimulation and pressure from the growing fetus could exert a stimulatory action. Amniotic fluid at term but not in mid-trimester has a strong stimulatory effect on the PG synthesis by decidua and myometrium, whereas the effect on amnion is not significant.

Besides $\mathrm{PGE}_{2}$ and $\mathrm{PGF}_{2} \alpha$, prostacyclin and to a lesser degree thromboxane and $\mathrm{PGD}_{2}$ are formed within the human uterus. Decidua and 
and myometrium produce large amounts of prostacyclin, and lesser amounts of $\mathrm{PGE}_{2}$ and $\mathrm{PGF} 2 \alpha$, whereas the amnion produces almost exclusively $\mathrm{PGE}_{2}$. Since $\mathrm{PGF}_{2} \alpha$ appears to be the most important prostanoid in the mechanism of labor, decidua may be more important than amnion as a site for prostanoid biosynthesis at the onset of labor. The fact that the $\mathrm{PGF}_{2 \alpha}$ production continues at a high rate long after the fetus and placenta with membranes are delivered also supports this hypothesis.

Both decidua and myometrium are highly vascularized tissues, and prostacyclin may be formed in their vascular compartments. This powerful vasodilating and anti-aggregatory agent may be of importance for the utero-placental blood flow. Thromboxane formation is restricted to the placenta, in which its synthesis under normal conditions is inhibited, but which may become of importance at delivery when the internal milieu suddenly changes. Placental thromboxane production may contribute to the hemostasis at placental separation. We have demonstrated that a major surge of PGE and F is released during the placental stage of labor, probably from the placenta and contribute to its expulsion.

Prostaglandins, relaxin, estrogen and progesterone all play a part in controlling the cervical changes that lead to increasing compliance toward the end of pregnancy. We have shown that myometrial sensitivity to oxytocin and the "ripeness" of the cervix increase in a parallel manner during the last seven days before parturition. oxytocin could therefore be the stimulus for the sporadic contractions that appear with increasing frequency in late pregnancy; in turn these may contribute to the rapid increase in cervical effacement in the last days before the onset of labor.

Adrenergic nerve activity results in the release of $\mathrm{PGE}_{2}$, at least in the spleen, heart and gut. If the same applies to the cervix, nerve activity could be of importance for cervical ripening. The remarkable release in response to vaginal examination and rupture of the membranes could perhaps also be mediated by nerve activity, at least partially.

Decidua is not an inert tissue but the site of synthesis of prostaglandins, prolactin and perhaps also relaxin. Decidua also has receptors for steroid hormones, glucocorticoids, and oxytocin. It has been postulated and widely quoted that prostaglandin synthesis in decidua is triggered by lysosomal instability, but others have failed to confirm this. Whatever triggers the synthesis of PGs in the decidua, of significance is the fact that the decidua is immediately adjacent to the myometrium, allowing for diffusion of humoral agents into the myometrial tissue without entering the vascular system. The amounts of PGs reaching the myometrium therefore need not be reflected in the blood levels.

Conclusions: Although less powerful oxytocics than oxytocin, PGE and $\mathrm{F}$ are produced within the uterus in close proximity to the site of action. The evidence suggests that oxytocin initiates labor and that the prostaglandins interact in the maintenance of labor, expulsion of fetus and placenta, and the post partum hemostasis. 